\title{
SPINOR EXTENDED LORENTZ-FORCE-LIKE EQUATIONS AS CONSEQUENCE OF A SPINORIAL STRUCTURE OF SPACE-TIME
}

\author{
J. Buitrago and S. Hajjawi \\ Department of Astrophysics of the University of La Laguna, \\ Avenida Francisco Sanchez, s/n, 38205, La Laguna, Tenerife, Spain*
}

(Dated: May 2006, revised November 2006)

\begin{abstract}
As formerly shown by one of us and briefly discussed in the Introduction below, the special relativistic dynamical equation of the Lorentz force type can be regarded as a consequence of a succession of space-time dependent infinitesimal Lorentz boosts and rotations. This insight indicates that the Lorentz-Force-like equation has a fundamental meaning in physics. In this paper we show how this result may be spinorially obtained starting out from the application of an infinitesimal element of $S L(2, C)$ to the individual spinors, which are here regarded as being more fundamental objects than four-vectors. In this way we get a set of new dynamical spinor equations inducing the extended Lorentz-Force-like equation in the Minkowski space-time and geometrically obtain the spinor form of the electromagnetic field-tensor. The term extended refers to the dynamics of some additional degrees of freedom that may be associated with the intrinsic spin, namely with the dynamics of three space-like mutually orthogonal four-vectors, all of them orthogonal to the linear four-momentum of the object under consideration which finally, in the particle's proper frame, are identified with the generators of $S U(2)$.

PACS numbers: $03.30 .+\mathrm{p}, 04.20 . \mathrm{Gz}$
\end{abstract}

\section{INTRODUCTION}

Some years ago one of us in $\underline{1}$ found that the Lorentz force equation may be regarded as a consequence of the infinitesimal transformation, within the Lorentz Group, which relates the four-momentum of the particle at (proper) times $\tau$ and $\tau+\delta \tau$ respectively. 
This result is obtained from purely geometrical considerations so one can conclude that, at the classical level, any force acting on a particle is restricted by the geometry of space-time to act in a very special, not arbitrary at all, way. Henceforth we shall call this fairly general result $\underline{7}$ the Geometrical Principle. Now, the motivation for the next section is that of re-interpreting this Principle as a consequence of the geometry of the Spinor Space $\mathcal{S}(2, \mathcal{C})$, in order to emphasize the idea that spinors are more fundamental objects than four-vectors and may thus be regarded as the elementary building blocks from which physical four-vectors are made of. We recall that any element $Q$ of $S L(2, C)$ acting on $\mathcal{S}(2, \mathcal{C})$, univocally defines a (proper) Lorentz transformation and reassume this idea by saying that the spinor spaces associated to any two distinct inertial observers are related through an element of $S L(2, C)$, which in turn induces a linear transformation on $\overline{\mathcal{S}(2, \mathcal{C})}$ through the element $\bar{Q}$ of $\overline{S L(2, C)} \underline{8}$. In ${ }^{\underline{1}}$ one starts out by considering that the four-momentum of a particle, which currently follows a trajectory in space-time, at proper times $\tau$ and $\tau+\delta \tau$ respectively, should be related through $\delta \Lambda^{\alpha}{ }_{\beta}$ by:

$$
p^{\alpha}(\tau+\delta \tau)=\delta \Lambda_{\beta}^{\alpha} p^{\beta}(\tau)
$$

where $\delta \Lambda^{\alpha}{ }_{\beta}$ is an infinitesimal element of the proper Lorentz group of transformations. It can be easily shown that this equation leads to a Lorentz-force-like equation and therefore, the Lorentz-Force is no more a semi-empirical result, but finds its theoretic justification within this context.

\section{SPINORIAL FORMULATION OF THE GEOMETRICAL PRINCIPLE}

As stated in the Introduction above, the components of any spinor associated with a particle following a trajectory in space-time at (proper) times $\tau$ and $\tau+\delta \tau$ respectively must be related through an infinitesimal element $\delta t_{B}{ }^{A}$ of $S L(2, C)$, so the key equation is (we adopt the standard spinor algebra notation as given $\mathrm{in}^{2}$ )

$$
\xi^{A}(\tau+\delta \tau)=\delta t_{B}{ }^{A} \xi^{B}
$$

where $\delta t_{B}{ }^{A}$ is of the form

$$
\delta t_{B}{ }^{A}=\left[e^{\frac{1}{2}(\delta \vec{\omega}+i \delta \vec{\theta}) \vec{\sigma}}\right] \equiv\left(e^{\delta \vec{\alpha} \vec{\sigma}}\right)_{B}{ }^{A}
$$


being $\delta \vec{\omega}=\left(\delta \omega_{1}, \delta \omega_{2}, \delta \omega_{3}\right), \delta \vec{\theta}=\left(\delta \theta_{1}, \delta \theta_{2}, \delta \theta_{3}\right)$ the infinitesimal parameters related to boosts and rotations respectively and $\vec{\sigma}=\left(\sigma_{1}, \sigma_{2}, \sigma_{3}\right)$ the vector containing the three Pauli spin matrices.

This starting point should lead us, via the Geometrical Principle, to the equations of motion of the particle in their spinorial form, as well as to a spinorial representation of the force-field acting on it, if our assertions are true. Since $\delta t_{B}{ }^{A}$ is infinitesimal, we expand the exponential term and keep only first-order-terms, so (1) becomes

$$
\xi^{A}(\tau+\delta \tau)=\xi^{B} \delta t_{B}{ }^{A}=\xi^{A}(\tau)+(\delta \vec{\alpha} \cdot \vec{\sigma})_{B}{ }^{A} \xi^{B}(\tau)
$$

being $(\delta \vec{\alpha} \cdot \vec{\sigma})_{B}{ }^{A}$ the matrix operator

$$
(\delta \vec{\alpha} \cdot \vec{\sigma})_{B}{ }^{A}=\left(\begin{array}{cc}
\delta \alpha_{3} & \delta \alpha_{1}+i \delta \alpha_{2} \\
\delta \alpha_{1}-i \delta \alpha_{2} & -\delta \alpha_{3}
\end{array}\right)
$$

which acts on the spinor $\xi^{B}$ transforming it infinitesimally. On the other side, if a change in velocity occurs, it should be proportional to the force-field acting on the particle and the lapse of proper time so we next define two fields $\vec{\epsilon}(x), \vec{\beta}(x)$ in such a way that

$$
\begin{aligned}
& \delta \vec{\omega}=\mathcal{K} \vec{\epsilon}(\bar{x}) \delta \tau, \\
& \delta \vec{\theta}=\mathcal{K} \vec{\beta}(\bar{x}) \delta \tau,
\end{aligned}
$$

being $\mathcal{K}$ some constant. In turn (2) can be manipulated to give

$$
\begin{gathered}
\delta \xi^{A} \equiv \xi^{A}(\tau+\delta \tau)-\xi^{A}(\tau) \\
=\xi^{B}(\tau)(\delta \vec{\alpha} \cdot \vec{\sigma})_{B}{ }^{A} \\
=\epsilon^{B C} \xi_{C}(\tau)(\delta \vec{\alpha} \cdot \vec{\sigma})_{B}{ }^{A} \\
=-\xi_{C}(\tau) \epsilon^{C B}(\delta \vec{\alpha} \cdot \vec{\sigma})_{B}{ }^{A}=\mathcal{K} \delta \tau \xi_{C}(\tau) \phi^{C A}=\mathcal{K} \delta \tau \phi^{A C} \xi_{C}(\tau) .
\end{gathered}
$$

In this way we have introduced the symmetric form $\phi^{A B}=-\epsilon^{A C}(\delta \vec{\alpha} \cdot \vec{\sigma})_{C}{ }^{B}$. From (4) one obtains

$$
\frac{d \xi^{A}}{d \tau} \equiv \dot{\xi}^{A}=\mathcal{K} \phi^{A B} \xi_{B}
$$

where $\phi^{A B}$ is explicitely given by

$$
\phi^{A B}=\frac{1}{2}\left(\begin{array}{cc}
-\left[\epsilon_{1}+\beta_{2}\right]+i\left[\epsilon_{2}-\beta_{1}\right] & \epsilon_{3}+i \beta_{3} \\
\epsilon_{3}+i \beta_{3} & {\left[\epsilon_{1}-\beta_{2}\right]+i\left[\beta_{1}+\epsilon_{2}\right]}
\end{array}\right) .
$$


Suppose now a massive particle currently acted on by an applied external field and let us see which equation of motion (5) leads to. Since the particle has non-null mass $m$, the spinorial representation of its four-momentum must be written as superposition of two null directions in the form

$$
p^{A A^{\prime}}=\pi^{A} \bar{\pi}^{A^{\prime}}+\eta^{A} \bar{\eta}^{A^{\prime}}
$$

resctricted by the condition

$$
p^{A A^{\prime}} p_{A A^{\prime}}=2\left|\pi^{A} \eta_{A}\right|^{2}=m^{2}
$$

Now, taking the derivative with respect to proper time and using (5) we get (we have set $\mathcal{K}=1$ for short)

$$
\begin{aligned}
& \dot{p}^{A A^{\prime}}=\dot{\pi}^{A} \bar{\pi}^{A^{\prime}}+\pi^{A} \dot{\bar{\pi}}^{A^{\prime}}+\dot{\eta}^{A} \bar{\eta}^{A^{\prime}}+\eta^{A} \dot{\bar{\eta}}^{A^{\prime}} \\
& =\phi^{A B} \pi_{B} \bar{\pi}^{A^{\prime}}+\bar{\phi}^{A^{\prime} B^{\prime}} \bar{\pi}_{B^{\prime}} \pi^{A}+\phi^{A B} \eta_{B} \bar{\eta}^{A^{\prime}}+\bar{\phi}^{A^{\prime} B^{\prime}} \bar{\eta}_{B^{\prime}} \eta^{A} \\
& =\epsilon^{A^{\prime} B^{\prime}} \phi^{A B} \pi_{B} \bar{\pi}_{B^{\prime}}+\epsilon^{A B} \bar{\phi}^{A^{\prime} B^{\prime}} \pi_{B} \bar{\pi}_{B^{\prime}}+\epsilon^{A^{\prime} B^{\prime}} \phi^{A B} \eta_{B} \bar{\eta}_{B^{\prime}}+\epsilon^{A B} \bar{\phi}^{A^{\prime} B^{\prime}} \eta_{B} \bar{\eta}_{B^{\prime}} \\
& =\left(\epsilon^{A B} \bar{\phi}^{A^{\prime} B^{\prime}}+\epsilon^{A^{\prime} B^{\prime}} \phi^{A B}\right) p_{B B^{\prime}} \\
& =F^{A A^{\prime} B B^{\prime}} p_{B B^{\prime}}
\end{aligned}
$$

where

$$
\epsilon^{A B} \bar{\phi}^{A^{\prime} B^{\prime}}+\epsilon^{A^{\prime} B^{\prime}} \phi^{A B}=F^{A A^{\prime} B B^{\prime}} .
$$

Spinorially, the general equation of motion is then given by, for $\mathcal{K}=1$

$$
\dot{p}^{A A^{\prime}}=F^{A A^{\prime} B B^{\prime}} p_{B B^{\prime}},
$$

so the index-lowered expression becomes

$$
\dot{p}_{A A^{\prime}}=F_{A A^{\prime} B B^{\prime}} p^{B B^{\prime}} .
$$

In the particular case of an external electromagnetic field, by replacing

$$
\begin{aligned}
& \vec{\epsilon}=\vec{E}, \\
& \vec{\beta}=\vec{B},
\end{aligned}
$$

and $\mathcal{K}=q / m$ we find out that (10) is just the spinorial representation of the Lorentz Force. Note, however, that in this way, the components of $F_{A A^{\prime} B B^{\prime}}$ are obtained from those of $\phi_{A B}$ 
not just as a spinorial transcription of the electromagnetic tensor, as it is usually done $\mathrm{e}^{\mathbf{5}}$. Instead, they have been here deduced from the geometric standpoint emphasized in (1), our starting point. On the other hand, in (11) we could have considered any other dynamical spinors instead of those associated to the four-momentum of a massive particle, so this result manifest how a spinorial structure hidden behind the minkowskian geometry of space-time at local level, makes the dynamical evolution of any physical four-vector to adopt the form of a Lorentz-Force- like equation.

In the case of an electromagnetic field and from (6), the components of $\phi^{A B}$ are related to the physical components of the field by

$$
\begin{gathered}
\phi^{00}=-\frac{1}{2}\left[\left(E_{x}+B_{y}\right)-i\left(E_{y}-B_{x}\right)\right], \\
\phi^{01}=\phi^{10}=\frac{1}{2}\left(E_{z}+i B_{z}\right) \\
\phi^{11}=\frac{1}{2}\left[\left(E_{x}-B_{y}\right)+i\left(E_{y}+B_{x}\right)\right]
\end{gathered}
$$

and the one-spinor equation of motion is re-writtten as

$$
\dot{\xi}^{A}=\frac{q}{m} \phi^{A B} \xi_{B}
$$

For the general case (no need to specify the nature of the force acting on the particle), we have

$$
\dot{\xi}^{A}=\mathcal{K} \phi^{A B} \xi_{B},
$$

being therefore the (spinorial) equation of motion for the four-momentum

$$
\dot{p}^{A A^{\prime}}=\mathcal{K} F^{A A^{\prime} B B^{\prime}} p_{B B^{\prime}}
$$

where $\mathcal{K}$ becomes a contant factor depending on some intrinsic feature of the particle and $\phi^{A B}$ is the spinorial representation of the force-field under consideration. Finally, the equivalent standard-tensorial (index-lowered) expression is

$$
\dot{p}_{\alpha}=\mathcal{K} F_{\alpha \beta} p^{\beta}
$$


with $F_{\alpha \beta}$ necessary obeying to

$$
F_{\alpha \beta}=-F_{\beta \alpha} .
$$

The last requirement is set by the (local) geometry of space-time and therefore the trajectory of a particle under the action of any force-field is restricted by the condition (17). Again, we remark that this idea constitutes the Geometrical Principle. The fact that $F_{\alpha \beta}$ must be anti-symmetric is a consequence of the minkowskian geometry, which in turn, in this new context, arises from a spinorial structure hidden behind it. On the other side, not only the form of the Lorentz-Force equation, as it is done, may be deduced: in the particular case that $F_{\alpha \beta}$ may be expressed in terms of a vectorial field $A_{\alpha}(x)$ as

$$
F_{\alpha \beta}=\partial_{\alpha} A_{\beta}-\partial_{\beta} A_{\alpha}
$$

Maxwell equations for free fields are an immediate consequence. EQUATION

\section{SPINOR EQUATIONS EQUIVALENT TO THE EXTENDED LORENTZ FORCE-LIKE EQUATION}

As we have already seen from (5) and the set of previous relations to (8), the dynamical evolution of the spinors $\pi^{A}, \eta^{A}$ (omitting for brevity the constant $k$ ) is given by

$$
\begin{aligned}
& \dot{\pi}^{A}=\phi^{A B} \pi_{B}, \\
& \dot{\eta}^{A}=\phi^{A B} \eta_{B} .
\end{aligned}
$$

Again, in the specific case of an electromagnetic field acting on the particle, these two relations are fully equivalent to the Lorentz Force. Note that the evolution for both $\pi^{A}$ and $\eta^{A}$ is given by the same differential form, so up to constant factors, they have the same analytic solution. On the other side, by carrying out

$$
\begin{gathered}
\left(\pi^{A} \eta_{A}\right)=\dot{\pi}^{A} \eta_{A}+\pi^{A} \dot{\eta}_{A} \\
=\phi^{A B} \pi_{B} \eta_{A}+\pi^{A} \phi^{B C} \eta_{C} \epsilon_{B A}=\phi^{A B} \pi_{B} \eta_{A}-\pi_{B} \phi^{B C} \eta_{C} \\
=\phi^{A B} \pi_{B} \eta_{A}-\phi^{B C} \pi_{B} \eta_{C}=\phi^{A B} \pi_{B} \eta_{A}-\phi^{C B} \pi_{B} \eta_{C}=0
\end{gathered}
$$


we see that $\pi^{A} \eta_{A}$ is a constant of motion in turn related, as we have already seen, to the mass of the particle by (7). This result has been already put forward by A. Bette and J. Buitrago ${ }^{3}$. Given the components of $\pi^{A}, \dot{\pi}^{A}$, referred to the spin basis $\left\{o^{A}=(0,1), i^{A}=(1,0)\right\}$ as

$$
\pi^{A}=\left(\begin{array}{c}
\pi^{0} \\
\pi^{1}
\end{array}\right), \quad \dot{\pi}^{A}=\left(\begin{array}{c}
\dot{\pi}^{0} \\
\dot{\pi}^{1}
\end{array}\right)
$$

we then obtain a set of two-coupled-differential equations, describing the dynamical evolution of the particle

$$
\begin{gathered}
\dot{\pi}^{A} o_{A}=\dot{\pi}^{1}=\phi^{A B} o_{A} \pi_{B}=\phi^{0 B} o_{0} \pi^{B}+\phi^{1 B} o_{1} \pi^{B}=\phi^{1 B} \pi_{B} \\
\dot{\pi}^{A} i_{A}=-\dot{\pi}^{0}=\phi^{A B} i_{A} \pi_{B}=\phi^{0 B} i_{0} \pi^{B}+\phi^{1 B} i_{1} \pi^{B}=-\phi^{0 B} \pi_{B} \\
\dot{\pi}^{1}=\phi^{10} \pi_{0}+\phi^{11} \pi_{1}=-\phi^{10} \pi^{1}+\phi^{11} \pi^{0} \\
\dot{\pi}^{0}=\phi^{00} \pi_{0}+\phi^{01} \pi_{1}=-\phi^{00} \pi^{1}+\phi^{01} \pi^{0}
\end{gathered}
$$

Consider now a four-vector spinorially represented by

$$
k^{A A^{\prime}}=\left(\alpha \pi^{A}+\beta \eta^{A}\right) \cdot\left(\gamma \bar{\pi}^{A^{\prime}}+\tau \bar{\eta}^{A^{\prime}}\right)
$$

with coefficients $\alpha, \beta, \gamma, \tau$ subject to the conditions

$$
\begin{aligned}
& \alpha \gamma=\overline{\alpha \gamma} \\
& \beta \tau=\overline{\beta \tau} \\
& \alpha \tau=\overline{\alpha \tau}
\end{aligned}
$$

whenever $k^{A A^{\prime}}$ is to represent a physical four-vector. Then with the equation of motion (10) it is easy to show that the evolution of $k^{A A^{\prime}}$ obeys to

$$
\dot{k}^{A A^{\prime}}=F^{A A^{\prime} B B^{\prime}} k_{B B^{\prime}}
$$

and, in particular, this holds for the following three space-like, dimensionless ${ }^{9}$ four-vectors spinorially defined ${ }^{3}$ by

$$
\begin{aligned}
s^{A A^{\prime}} & =\frac{\pi^{A} \bar{\pi}^{A^{\prime}}-\eta^{A} \bar{\eta}^{A^{\prime}}}{m}, \\
v^{A A^{\prime}} & =\frac{\pi^{A} \bar{\eta}^{A^{\prime}}+\eta^{A} \bar{\pi}^{A^{\prime}}}{m},
\end{aligned}
$$




$$
w^{A A^{\prime}}=\frac{i\left(\pi^{A} \bar{\eta}^{A^{\prime}}-\eta^{A} \bar{\pi}^{A^{\prime}}\right)}{m},
$$

which fullfill the conditions

$$
\begin{gathered}
s^{a} s_{a}=v^{a} v_{a}=w^{a} w_{a}=-1, \\
s^{a} p_{a}=s^{a} v_{a}=s^{a} w_{a}=0, \\
v^{a} p_{a}=v^{a} w_{a}=0, w^{a} p_{a}=0 .
\end{gathered}
$$

and constitute, together with $p^{A A^{\prime}}$ an orthogonal tetrad. The ordinary physical components of these vectors are obtained in the usual way by

$$
\begin{aligned}
& s^{A A^{\prime}} i_{A} \bar{i}_{A^{\prime}}=\frac{s^{0}+s^{3}}{\sqrt{2}}, \quad s^{A A^{\prime}} O_{A} \bar{o}_{A^{\prime}}=\frac{s^{0}-s^{3}}{\sqrt{2}} \\
& s^{A A^{\prime}} o_{A} \bar{i}_{A^{\prime}}=\frac{s^{1}+i s^{2}}{\sqrt{2}}, \quad s^{A A^{\prime}} \bar{o}_{A^{\prime}} i_{A}=\frac{s^{1}-i s^{2}}{\sqrt{2}} \\
& v^{A A^{\prime}} i_{A} \bar{i}_{A^{\prime}}=\frac{v^{0}+v^{3}}{\sqrt{2}}, \quad v^{A A^{\prime}} o_{A} \bar{o}_{A^{\prime}}=\frac{v^{0}-v^{3}}{\sqrt{2}} \\
& v^{A A^{\prime}}{ }_{O_{A}} \bar{i}_{A^{\prime}}=\frac{v^{1}+i v^{2}}{\sqrt{2}}, \quad v^{A A^{\prime}} \bar{o}_{A^{\prime}} i_{A}=\frac{v^{1}-i v^{2}}{\sqrt{2}} \\
& w^{A A^{\prime}} i_{A} \bar{i}_{A^{\prime}}=\frac{w^{0}+w^{3}}{\sqrt{2}}, \quad w^{A A^{\prime}} o_{A} \bar{o}_{A^{\prime}}=\frac{w^{0}-w^{3}}{\sqrt{2}} \\
& w^{A A^{\prime}} o_{A} \bar{i}_{A^{\prime}}=\frac{w^{1}+i w^{2}}{\sqrt{2}}, \quad w^{A A^{\prime}} \bar{o}_{A^{\prime}} i_{A}=\frac{w^{1}-i w^{2}}{\sqrt{2}}
\end{aligned}
$$


In the particle's (instantaneous) proper frame, these four-vectors become purely spacial (they will be further identified with the components of an intrinsic angular momentum) and so any linear combination $J^{\alpha}$ of them, which should evolve at any arbitrary frame, according to

$$
\dot{J}_{\alpha}=F_{\alpha \beta} J^{\beta}
$$

\section{INTRINSIC DEGREES OF FREEDOM OF MASSIVE AND MASSLESS PARTICLES}

So far, we have seen how the requirement within this formalism of expressing the fourmomentum of a massive particle as superposition of two null-directions, as

$$
p^{A A^{\prime}}=\pi^{A} \bar{\pi}^{A^{\prime}}+\eta^{A} \bar{\eta}^{A^{\prime}}
$$

lead us, in a natural way, to define the set of three dynamical additional four-vectors

$$
\begin{gathered}
s^{A A^{\prime}}=\frac{\pi^{A} \bar{\pi}^{A^{\prime}}-\eta^{A} \bar{\eta}^{A^{\prime}}}{m}, \\
v^{A A^{\prime}}=\frac{\pi^{A} \bar{\eta}^{A^{\prime}}+\eta^{A} \bar{\pi}^{A^{\prime}}}{m}, \\
w^{A A^{\prime}}=\frac{i\left(\pi^{A} \bar{\eta}^{A^{\prime}}-\eta^{A} \bar{\pi}^{A^{\prime}}\right)}{m},
\end{gathered}
$$

which, together with $p^{A A^{\prime}}$, form a tetrad that appears to be something inherent to the particle under consideration, merely as a consequence of following a trajectory in spacetime. In the previous Section we have already pointed out that they may be associated to internal degrees of freedom and in the next one, we shall see that they do behave like components of an intrinsic angular momentum. Here we shall try to get some general information about them by analyzing their components in the (instantaneous) rest frame, for intrinsic properties such as the spin make sense only in this inertial frame. Throughout this section, and just for brief notation, any quantity is understood to be referred to the particle's proper frame, in which the conditions

$$
\begin{gathered}
\vec{p}=\overrightarrow{0}, \\
s^{0}=v^{0}=w^{0}=0 .
\end{gathered}
$$


must hold. By expressing $\pi^{A}, \eta^{A}$ in a general way as

$$
\pi^{A}=\left(\begin{array}{c}
\left|\pi^{0}\right| e^{i \phi_{0}} \\
\left|\pi^{1}\right| e^{i \phi_{1}}
\end{array}\right), \eta^{A}=\left(\begin{array}{c}
\left|\eta^{0}\right| e^{i \xi_{0}} \\
\left|\eta^{1}\right| e^{i \xi_{1}}
\end{array}\right)
$$

and from the conditions

$$
\begin{aligned}
& p_{3}=0 \Longrightarrow\left|\pi^{0}\right|^{2}+\left|\eta^{0}\right|^{2}-\left|\pi^{1}\right|^{2}-\left|\eta^{1}\right|^{2}=0, \\
& s_{0}=0 \Longrightarrow\left|\pi^{0}\right|^{2}-\left|\eta^{0}\right|^{2}+\left|\pi^{1}\right|^{2}-\left|\eta^{1}\right|^{2}=0
\end{aligned}
$$

we get

$$
\begin{aligned}
& \left|\eta^{0}\right|^{2}=\left|\pi^{1}\right|^{2} \\
& \left|\eta^{0}\right|^{2}=\left|\pi^{1}\right|^{2}
\end{aligned}
$$

so $\eta^{A}$ adopts, in this particular frame, the form:

$$
\eta^{A}=\left(\begin{array}{c}
\left|\pi^{1}\right| e^{i \xi_{0}} \\
\left|\pi^{0}\right| e^{i \xi_{1}}
\end{array}\right)
$$

and the components $v^{00^{\prime}}, v^{11^{\prime}}, w^{11^{\prime}}$ are then given by

$$
\begin{gathered}
\left\{\begin{array}{c}
v^{00^{\prime}}=\frac{2}{m}\left|\pi^{0} \pi^{1}\right| \cos \left(\phi_{0}-\xi_{0}\right) \\
v^{11^{\prime}}=\frac{2}{m}\left|\pi^{0} \pi^{1}\right| \cos \left(\phi_{1}-\xi_{1}\right)
\end{array}\right\} \Longrightarrow \cos \left(\phi_{0}-\xi_{0}\right)+\cos \left(\phi_{1}-\xi_{1}\right)=0 \\
\left\{\begin{array}{c}
w^{00^{\prime}}=-\frac{2}{m}\left|\pi^{0} \pi^{1}\right| \sin \left(\phi_{0}-\xi_{0}\right) \\
w^{11^{\prime}}=-\frac{2}{m}\left|\pi^{0} \pi^{1}\right| \cos \left(\phi_{1}-\xi_{1}\right)
\end{array}\right\} \Longrightarrow \sin \left(\phi_{0}-\xi_{0}\right)+\cos \left(\phi_{1}-\xi_{1}\right)=0
\end{gathered}
$$

The conditions given in (39), (40) are necessary in order to guarantee that $v^{0}=w^{0}=0$, so we find that the phase factors $\phi_{0}, \phi_{1}, \xi_{0}, \xi_{1}$ must be related by

$$
\left(\phi_{1}-\xi_{1}\right)=\left(\phi_{0}-\xi_{0}\right)+(2 n+1) \pi
$$

or equivalently

$$
\left(\phi_{1}-\phi_{0}\right)=\left(\xi_{1}-\xi_{0}\right)+(2 n+1) \pi .
$$


This, together with the conditions given in (36) and (37), leads to the automatic fullfillment of

$$
\vec{s} \cdot \vec{v}=\vec{s} \cdot \vec{w}=\vec{v} \cdot \vec{w}=0
$$

On the other side, in the particle's proper frame, the two null-directions $\pi^{A} \bar{\pi}^{A^{\prime}}, \eta^{A} \bar{\eta}^{A^{\prime}}$ must appear to have opposite momentum in order to satisfy the condition above mentioned $(\vec{p}=$ $\overrightarrow{0})$. Therefore, the energy associated with each of them must be the same and subsequently equal to $m / 2$, a result that may be checked through the relations

$$
\begin{gathered}
\left\{\begin{array}{c}
2 p_{\pi}^{0}=\sqrt{2}\left[\left|\pi^{0}\right|^{2}+\left|\pi^{1}\right|^{2}\right] \\
2 p_{\eta}^{0}=\sqrt{2}\left[\left|\pi^{0}\right|^{2}+\left|\pi^{1}\right|^{2}\right]
\end{array}\right\} \Longrightarrow p_{\pi}^{0}=p_{\eta}^{0}=\omega, \\
\left\{\begin{array}{l}
2 p_{\pi}^{3}=\sqrt{2}\left[\left|\pi^{0}\right|^{2}-\left|\pi^{1}\right|^{2}\right] \\
2 p_{\eta}^{3}=\sqrt{2}\left[\left|\pi^{1}\right|^{2}-\left|\pi^{0}\right|^{2}\right]
\end{array}\right\} \Longrightarrow p_{\pi}^{3}=-p_{\eta}^{3}, \\
\left\{\begin{array}{l}
p_{\pi}^{1}=\sqrt{2}\left|\pi^{0} \pi^{1}\right| \cos \left(\phi_{0}-\phi_{1}\right) \\
p_{\eta}^{1}=\sqrt{2}\left|\pi^{0} \pi^{1}\right| \cos \left(\xi_{0}-\xi_{1}\right)
\end{array}\right\} \Longrightarrow p_{\pi}^{1}=-p_{\eta}^{1}, \\
\left\{\begin{array}{l}
p_{\pi}^{2}=\sqrt{2}\left|\pi^{0} \pi^{1}\right| \sin \left(\phi_{0}-\phi_{1}\right) \\
p_{\eta}^{2}=\sqrt{2}\left|\pi^{0} \pi^{1}\right| \sin \left(\xi_{0}-\xi_{1}\right)
\end{array}\right\} p_{\pi}^{2}=-p_{\eta}^{2},
\end{gathered}
$$

where $\omega$ is related to the rest-energy of the particle by

$$
2 \omega=m \text {. }
$$

Now, in terms of $\omega$ the dimensionless, normalized four-vectors $s^{A A^{\prime}}, v^{A A^{\prime}}, w^{A A^{\prime}}$ are re-written as 


$$
\begin{gathered}
s^{A A^{\prime}}=\frac{\pi^{A} \bar{\pi}^{A^{\prime}}-\eta^{A} \bar{\eta}^{A^{\prime}}}{2 \omega}, \\
v^{A A^{\prime}}=\frac{\pi^{A} \bar{\eta}^{A^{\prime}}+\eta^{A} \bar{\pi}^{A^{\prime}}}{2 \omega}, \\
w^{A A^{\prime}}=i \frac{\left(\pi^{A} \bar{\eta}^{A^{\prime}}-\eta^{A} \bar{\pi}^{A^{\prime}}\right)}{2 \omega},
\end{gathered}
$$

Note now that both, $v^{A A^{\prime}}$ and $w^{A A^{\prime}}$, are Lorentz-orthogonal to simultaneously $\pi^{A} \bar{\pi}^{A^{\prime}}, \eta^{A} \bar{\eta}^{A^{\prime}}$, which spinorially represent null-directions (e.g. photons) and so the relations

$$
\begin{gathered}
k^{\mu} k_{\mu}=0, \\
k^{\mu} v_{\mu}=k^{\mu} w_{\mu}=0, \\
v^{\mu} w_{\mu}=0,
\end{gathered}
$$

where $k^{\mu}$ represents the four-momentum either of $\pi^{A} \bar{\pi}^{A^{\prime}}$ or $\eta^{A} \bar{\eta}^{A^{\prime}}$, hold. The four-vectors $v^{\alpha}, w^{\alpha}$ may be then identified with polarization vectors for the null-direction $k^{\mu}$ in the Lorentz-gauge, for which the condition imposed is expressed as

$$
k^{\mu} \epsilon_{\mu}(\vec{k}, \lambda)=0
$$

This allows, as we know, a set of two linearly-independent such vectors $(\lambda=1,2)$. In fact, for any null-direction spinorially represented as $\pi^{A} \bar{\pi}^{A^{\prime}}$, any set of two four-vectors of the form

$$
\begin{aligned}
v^{A A^{\prime}} & \propto\left[\pi^{A} \bar{\xi}^{A^{\prime}}+\xi^{A} \bar{\pi}^{A^{\prime}}\right], \\
w^{A A^{\prime}} & \propto i\left[\pi^{A} \bar{\xi}^{A^{\prime}}-\xi^{A} \bar{\pi}^{A^{\prime}}\right],
\end{aligned}
$$

satisfies the relations (47) for any spinor $\xi^{A}$ non- proportional to $\pi^{A}$. Now, by choosing $\xi^{A}$ as the counterpart of $\pi^{A}$ giving rise to the null direction $\xi^{A} \bar{\xi}^{A^{\prime}}$ with opposite spacial momentum to $\pi^{A} \bar{\pi}^{A^{\prime}}$, we have that

$$
\vec{\epsilon}(\vec{k}, \lambda) \cdot \vec{k}=0
$$

This is then fully equivalent to choose the well-known gauge transformation of second kind known as the Coulomb gauge. This is precisely the behaviour found in the rest frame with respect to $\eta^{A}$, as we saw in the relations (43)-(45). In this frame the null directions $\pi^{A} \bar{\pi}^{A^{\prime}}, \eta^{A} \bar{\eta}^{A^{\prime}}$ together with the associated four-vectors $v^{A A^{\prime}}, w^{A A^{\prime}}$ do therefore represent null-mass particles of spin 1 and energy $\omega=m / 2$. Finally, the four-vector $s^{A A^{\prime}}$ becomes 
parallel to $\vec{p}_{\pi}$, as it is shown in the relations

$$
\begin{gathered}
\left\{\begin{array}{c}
s^{00^{\prime}}=\frac{\left|\pi^{0}\right|^{2}-\left|\pi^{1}\right|^{2}}{2 \omega} \\
s^{11^{\prime}}=\frac{\left|\pi^{1}\right|^{2}-\left|\pi^{0}\right|^{2}}{2 \omega}
\end{array}\right\} \Longrightarrow s^{00^{\prime}}=0, s^{3}=\frac{1}{\sqrt{2}} \frac{\left[\left|\pi^{0}\right|^{2}-\left|\pi^{1}\right|^{2}\right]}{\omega}, \\
s^{01^{\prime}}=\frac{\left|\pi^{0} \pi^{1}\right|}{2 \omega}\left(e^{i\left(\phi_{0}-\phi_{1}\right)}-e^{i\left(\xi_{0}-\xi_{1}\right)}\right) \Longrightarrow\left\{\begin{array}{l}
s^{1}=\frac{1}{\sqrt{2}}\left|\pi^{0} \pi^{1}\right| \cos \left(\phi_{0}-\phi_{1}\right) \omega \\
s^{2}=\frac{1}{\sqrt{2}}\left|\pi^{0} \pi^{1}\right| \sin \left(\phi_{0}-\phi_{1}\right) \omega
\end{array}\right\} .
\end{gathered}
$$

FIELD

\section{EVOLUTION OF INTRINSIC SPIN UNDER AN HOMOGENEOUS CONSTANT MAGNETIC FIELD}

Suppose a massive $(m=1)$, charged particle in the presence of and external, homogeneous, constant magnetic field directed along the $z$-axis. By applying the stardard Lorentz Force equation and solving the first order coupled system for the evolution of the momentum we get

$$
\begin{gathered}
p_{z}(\tau)=\text { constant }, \\
p_{x}(\tau)=A_{1} \cos (q B \tau)+A_{2} \sin (q B \tau), \\
p_{y}(\tau)=A_{2} \cos (q B \tau)-A_{1} \sin (q B \tau),
\end{gathered}
$$

where $A_{1}$ and $A_{2}$ are to be determined from the initial conditions. In this case, the equations to solve are

$$
\begin{gathered}
\dot{\pi}^{0}=-i \frac{q B}{2} \pi^{0}, \\
\dot{\pi}^{1}=i \frac{q B}{2} \pi^{1},
\end{gathered}
$$

(the same holds for $\dot{\eta}^{A}$ ). Note that, in this case, the equations become simpler than in the traditional formulation and will also give the evolution of the intrinsic spin. This follows from the fact that, according to the geometrical interpretation of the Lorentz Force ${ }^{\underline{1}}$, the $\vec{B}$ components are related to rotations; hence to the $S U(2)$ subgroup of $S L(2, C)$ related to 
spin. The solution to these equations is given by

$$
\begin{gathered}
\pi^{0}(\tau)=\pi^{0}(\tau=0) \exp \left(-i \frac{q B}{2} \tau\right), \\
\pi^{1}(\tau)=\pi^{1}(\tau=0) \exp \left(i \frac{q B}{2} \tau\right), \\
\eta^{0}(\tau)=\eta^{0}(\tau=0) \exp \left(-i \frac{q B}{2} \tau\right), \\
\eta^{1}(\tau)=\eta^{1}(\tau=0) \exp \left(i \frac{q B}{2} \tau\right),
\end{gathered}
$$

and therefore

$$
\begin{gathered}
p^{00^{\prime}}=\left|\pi^{0}(\tau=0)\right|^{2}+\left|\eta^{0}(\tau=0)\right|^{2}=\text { constant } \\
p^{01^{\prime}}=\pi^{0}(\tau=0) \bar{\pi}^{1^{\prime}}(\tau=0) \exp (-i q B \tau)+\eta^{0}(\tau=0) \bar{\eta}^{1^{\prime}}(\tau=0) \exp (-i q B \tau)=A(\tau=0) \exp (-i q B \tau), \\
p^{11^{\prime}}=\left|\pi^{1}(\tau=0)\right|^{2}+\left|\eta^{1}(\tau=0)\right|^{2}=\text { constant }
\end{gathered}
$$

where $A(\tau=0)$ is a complex number whose value is to be determined from the initial conditions, so $p^{01^{\prime}}$ can be written in the form

$$
p^{01^{\prime}}(\tau)=A_{1} \cos (q B \tau)+A_{2} \sin (q B \tau)+i\left[A_{2} \cos (q B \tau)-A_{1} \sin (q B \tau)\right]
$$

being $A_{1}=\operatorname{Re}\{A(\tau=0)\}$ and $A_{2}=\operatorname{Im}\{A(\tau=0)\}$. We get then for the components of the four-momentum:

$$
\begin{gathered}
\left\{\begin{array}{c}
\sqrt{2} p^{00^{\prime}}=E+p_{z}=\text { constant } \\
\sqrt{2} p^{11^{\prime}}=E-p_{z}=\text { constant }
\end{array}\right\} \Longrightarrow E, p_{z}=\text { constant }, \\
\sqrt{2} p^{01^{\prime}}=p_{x}+i p_{y} \Longrightarrow\left\{\begin{array}{l}
p_{x}=\sqrt{2}\left[A_{1} \cos (q B \tau)+A_{2} \sin (q B \tau)\right] \\
p_{y}=\sqrt{2}\left[A_{2} \cos (q B \tau)-A_{1} \sin (q B \tau)\right]
\end{array}\right\}
\end{gathered}
$$


which, as expected, coincides with the well-known solutions. For the time evolution of the four-vectors $s^{a}, v^{a}, w^{a}$ we find

$$
\begin{gathered}
\left\{\begin{array}{c}
\sqrt{2} s^{00^{\prime}}=\frac{\sqrt{2}}{m}\left[\left|\pi^{0}(\tau=0)\right|^{2}-\left|\eta^{0}(\tau=0)\right|^{2}\right]=s^{0}+s^{z} \\
\sqrt{2} s^{11^{\prime}}=\frac{\sqrt{2}}{m}\left[\left|\pi^{1}(\tau=0)\right|^{2}-\left|\eta^{1}(\tau=0)\right|^{2}\right]=s^{0}-s^{z}
\end{array}\right\} \Longrightarrow s^{0}, s^{z}=\text { constant }, \\
\left\{\begin{array}{l}
\sqrt{2} v^{00^{\prime}}=\frac{\sqrt{2}}{m}\left[\pi^{0}(\tau=0) \bar{\eta}^{0}(\tau=0)+c . c .\right]=v^{0}+v^{z} \\
\sqrt{2} v^{11^{\prime}}=\frac{\sqrt{2}}{m}\left[\pi^{1}(\tau=0) \bar{\eta}^{1}(\tau=0)+c . c .\right]=v^{0}-v^{z}
\end{array}\right\} \Longrightarrow v^{0}, v^{z}=\text { constant }, \\
\left\{\begin{array}{l}
\sqrt{2} w^{00^{\prime}}=\frac{\sqrt{2}}{m}\left[i \pi^{0}(\tau=0) \bar{\eta}^{0}(\tau=0)+c . c .\right]=w^{0}+w^{z} \\
\sqrt{2} w^{11^{\prime}}=\frac{\sqrt{2}}{m}\left[i \pi^{1}(\tau=0) \bar{\eta}^{1}(\tau=0)+c . c .\right]=w^{0}-w^{z}
\end{array}\right\} \Longrightarrow w^{0}, w^{z}=\text { constant }, \\
\sqrt{2} s^{01^{\prime}}=s_{x}+i s_{y}=B(\tau=0) e^{(-q B \tau)} \Longrightarrow\left\{\begin{array}{l}
s_{x}=B_{1} \cos (q B \tau)+B_{2} \sin (q B \tau) \\
s_{y}=B_{2} \cos (q B \tau)-B_{1} \sin (q B \tau)
\end{array}\right\} \\
\sqrt{2} v^{01^{\prime}}=v_{x}+i v_{y}=C(\tau=0) e^{(-q B \tau)} \Longrightarrow\left\{\begin{array}{l}
w_{x}=D_{1} \cos (q B \tau)+D_{2} \sin (q B \tau) \\
v_{x}=C_{1} \cos (q B \tau)+C_{2} \sin (q B \tau) \\
v_{y}=C_{2} \cos (q B \tau)-C_{1} \sin (q B \tau)
\end{array}\right\}
\end{gathered}
$$

If the initial conditions are such that $p_{x}(\tau=0)=p_{y}(\tau=0)=0$ the particle keeps on moving with constant velocity along the $z$-axis and the (orbital) angular momentum is therefore null. However, if the particle is to have an intrinsic angular momentum, although no Thomas precession occurs, we should expect its intrinsic magnetic moment to precess around the $z$-axis, due to the presence of the magnetic field. This is precisely the behaviour found for $\vec{s}, \vec{v}, \vec{w}$ : they do precess due solely to the presence of the applied magnetic field and can be therefore identified with the components of an intrinsic angular momentum. To 
analyze the situation in the rest frame of the particle, we set $\vec{p}=0$. Then, we find that the temporal evolution of the spinors in this frame is

$$
\pi^{A}(\tau)=\left(\begin{array}{c}
\left|\pi^{0}\right|(\tau=0) e^{i \phi_{0}} e^{i \frac{q B \tau}{2}} \\
\left|\pi^{1}\right|(\tau=0) e^{i \phi_{1}} e^{i \frac{q B \tau}{2}}
\end{array}\right), \eta^{A}(\tau)=\left(\begin{array}{c}
\left|\pi^{1}\right|(\tau=0) e^{i \xi_{0}} e^{i \frac{q B \tau}{2}} \\
\left|\pi^{0}\right|(\tau=0) e^{i \xi_{1}} e^{i \frac{q B \tau}{2}}
\end{array}\right)
$$

where the phase-factors $\phi_{0}, \phi_{1}, \xi_{0}, \xi_{1}$ are related by (41). For the momenta of the null directions associated to massless particles in this inertial frame we find

$$
\begin{gathered}
\vec{p}_{\pi}=\left(\frac{\left|\pi^{0} \pi^{1}\right|}{\sqrt{2}} \cos (q B \tau+\delta \phi), \frac{\left|\pi^{0} \pi^{1}\right|}{\sqrt{2}} \sin (q B \tau+\delta \phi), \sqrt{2}\left[\left|\pi^{0}\right|^{2}-\left|\pi^{1}\right|^{2}\right]\right), \\
\vec{p}_{\eta}=-\left(\frac{\left|\pi^{0} \pi^{1}\right|}{\sqrt{2}} \cos (q B \tau+\delta \phi), \frac{\left|\pi^{0} \pi^{1}\right|}{\sqrt{2}} \sin (q B \tau+\delta \phi), \sqrt{2}\left[\left|\pi^{0}\right|^{2}-\left|\pi^{1}\right|^{2}\right]\right),
\end{gathered}
$$

with $\delta \phi=\left(\phi_{0}-\phi_{1}\right)$.

We see in (63) and (64) that the $z$-component remains constant in time, while the $x$ - and $y$ components do evolve. This causes, in this particular case, the rotation of $\vec{p}_{\pi}$ and $\vec{p}_{\eta}$ around the $z$-axis. With use of the equations (13), (14), (15) one obtains (note that $\vec{s}$ is aligned with $\left.\vec{p}_{\pi}\right)$

$$
\begin{gathered}
\vec{s}=\frac{\vec{p}_{\pi}}{m}, \\
v_{x}=\frac{\sqrt{2}}{m}\left\{\left|\pi^{0}\right|^{2} \cos \left(q B \tau+\phi_{0}-\xi_{1}\right)-\left|\pi^{1}\right|^{2} \cos \left(q B \tau+\xi_{0}-\phi_{1}\right)\right\} \\
v_{y}=\frac{\sqrt{2}}{m}\left\{\left|\pi^{0}\right|^{2} \sin \left(q B \tau+\phi_{0}-\xi_{1}\right)-\left|\pi^{1}\right|^{2} \sin \left(q B \tau+\xi_{0}-\phi_{1}\right)\right\}, \\
w_{x}=\frac{\sqrt{2}}{m}\left\{\left|\pi^{1}\right|^{2} \sin \left(q B \tau+\xi_{0}-\phi_{1}\right)-\left|\pi^{0}\right|^{2} \sin \left(q B \tau+\phi_{0}-\xi_{1}\right)\right\} \\
w_{y}=\frac{\sqrt{2}}{m}\left\{\left|\pi^{0}\right|^{2} \cos \left(q B \tau+\phi_{0}-\xi_{1}\right)-\left|\pi^{1}\right|^{2} \cos \left(q B \tau+\xi_{0}-\phi_{1}\right)\right\}, \\
w_{z}=-\frac{2 \sqrt{2}}{m}\left|\pi^{0} \pi^{1}\right| \sin \left(\phi_{0}-\xi_{0}\right) .
\end{gathered}
$$

Notice that $\vec{s}, \vec{v}, \vec{w}$ keep instantaneously, at any proper time $\tau$, orthogonal to each other while undergoing the precession around the $z$-axis. This will also happen to any vector formed as a linear combination of them. However, this point needs further clarification which will be the subject on next section. 


\section{SPINORIAL REPRESENTATION OF AN INTRINSIC SPIN}

So far, we have seen how the three four-vectors $s^{A A^{\prime}}, v^{A A^{\prime}}, w^{A A^{\prime}}$ behave in the particle's proper frame as components of an intrinsic angular momentum vector under the action of an applied external magnetic field. This fact seems to indicate that these four-vectors are, somehow, related to the intrinsic spin of the particle. To elucidate further this point, we switch off the magnetic field and recall that, in the particle's proper frame, the spinors $\pi^{A} \bar{\pi}^{A^{\prime}}$ and $\eta^{A} \bar{\eta}^{A^{\prime}}$ do represent null-directions having the same energy and opposite spacial momentum. However, the direction of $\vec{p}_{\pi}$ is absolutely arbitrary and we can take it, for instance, along the $z$-direction. With this we have that

$$
\pi^{A}=\left(\begin{array}{c}
\pi^{0} \\
0
\end{array}\right), \quad \eta^{A}=\left(\begin{array}{c}
0 \\
\eta^{1}
\end{array}\right)
$$

With this representation, $\pi^{A} \pi^{A^{\prime}}$ represents a massless particle moving along the $z-$ direction, in positive sense. Next, the condition of having the same energy imposes $\left|\eta^{1}\right|=$ $\left|\pi^{0}\right|$; with this, the more general expression for both $\pi^{A}, \eta^{A}$ is

$$
\pi^{A}=\left|\pi^{0}\right|\left(\begin{array}{c}
e^{i \phi_{0}} \\
0
\end{array}\right), \quad \eta^{A}=\left|\pi^{0}\right|\left(\begin{array}{c}
0 \\
e^{i \xi_{1}}
\end{array}\right)
$$

Again there is arbitrariness with respect to the phase factors $\phi_{0}, \xi_{1}$. We shall partially remove it by setting $\xi_{1}=\phi_{0}$. Next, remember that, although $s^{A A^{\prime}}, v^{A A^{\prime}}, w^{A A^{\prime}}$ are four-vectors, they have also an associated matrix representation, in such a way that its determinant coincides with the Lorentz-norm of the corresponding four-vector. Taking into account the condition (7), these associated matrices are given by

$$
s^{A A^{\prime}}=\left(\begin{array}{cc}
1 & 0 \\
0 & -1
\end{array}\right), \quad v^{A A^{\prime}}=\left(\begin{array}{ll}
0 & 1 \\
1 & 0
\end{array}\right), \quad w^{A A^{\prime}}=\left(\begin{array}{cc}
0 & i \\
-i & 0
\end{array}\right) .
$$

The reader will immediately recognize the three Pauli-spin matrices 10 , which represent the intrinsic angular momentum of a one-half spin massive particle. Since equation (70) correspond to four-vectors with null-time component, following the steps of Quantum Mechanics, we could define the spin operator of the particle, at its rest-frame, as

$$
\vec{S}=\frac{1}{2}\left(s^{A A^{\prime}}, v^{A A^{\prime}}, w^{A A^{\prime}}\right)
$$


and related through a Lorentz transformation to any other inertial frame.

\section{FINAL REMARKS}

According to the principle presented in ${ }^{\underline{1}}$ and summarized in Section $I$, the form of the classical equations of motion can be regarded as a consequence of the geometry of the complex two-dimensional Spin Space which sets in turn the local geometry of space-time to be minkowskian. In this context, spinors are more fundamental objects than four-vectors and, as seen in IV, V and VI, are able to describe the intrinsic spin of massless particles and also the intrinsic spin $1 / 2$ of massive ones . The before mentioned principle lead us to a set of new spinorial differential equations describing the dynamics of a massive spinning object, since not only the evolution of the world trajectories are contained in these equations, but are also susceptible of describing new degrees of freedom associated to its intrinsic spin which turns out to be the corresponding to spin one-half particles. Finally we would like to point out that the space-time dynamics described in this way are performed entirely in spinor space and do not make explicit use of the space-time coordinates that become secondary constructions. In this sense, our study differ from the twistor approach (especially in its two-twistor version, see e.g. $\cdot \underline{6})$ which make use of the space-time coordinates.

\section{Acknowledgment}

We would like specially thank Doctor Andreas Bette (deceased in May 2005) of the Royal Institute of Technology (Sweden), for inspiring some of the ideas here developed.

* Electronic address: jgb@ll.iac.es

1 Buitrago Jesús, 1995, Electromagnetic force and geometry of Minkowskian spacetime., Eur. J. Phys. 16, 113-118.

2 Penrose Roger and Rindler Wolfgang, (1984) and (1986). Spinors and Space-Time, Cambridge Monographs on Mathematical Physics vol.1 and 2, Cambridge University Press, Cambridge. 
3 A. Bette and J. Buitrago, XIX-th Max Born Symposium: Fundamental Interactions and Twistor-like Methods. American Institute of Physics Conference Proceedings No. 767, pp. 28-44 (205).

4 Bette Andreas, 2003. Twistors, Special relativity, conformal symmetry and minimal coupling a review., Reporte de Investigacion No. 26, Septiembre 2003, Universidad de Sonora, Division de Ciencias Exactas y Naturales, Rosales y Blvd. Luis Encinas J., Edif. 3K1, 83000 Hermosillo, Sonora, Mexico.

5 Stewart John, (1990). Advanced General Relativity, Cambridge University Press, Cambridge Monographs on Mathematical Physics.

${ }^{6}$ Bette Andreas et al. (2004). Massive Relativistic Particle Model with Spin and Electric Charge from Two-Twistor Dynamics, Phys. Lett. B, 595, 491-497.

7 The Geometrical Principle is, in fact, not only applicable to the four-momenta, but to any four-vector representing any physical magnitude.

8 This is better understood if we recall that $\mathcal{M}_{4}$ is a sub-space of the four-complex-dimensional vector-space $\mathcal{S}(2, \mathcal{C}) \otimes \overline{\mathcal{S}(2, \mathcal{C})}$.

9 Note that in natural units the angular momentum is also dimensionless.

10 Although the usual representation of the Pauli-spin-matrices sets $\epsilon_{123}=1$, due to the global sign chose for $w^{A A^{\prime}}$, the representation here given corresponds to $\epsilon_{123}=-1$. A global sign however does not affect previous or further results. 